\section{Can analysis of aortic calcification be used to predict risk of hip fracture?}

Population-based cohort studies have previously shown a correlation between bone loss and progression of aortic calcification, as well as an inverse relationship between bone mass and incidence of coronary heart disease, in postmenopausal women. Other studies have found a correlation between cardiovascular disease-associated mortality and low bone mineral density, increased bone loss and vertebral fracture.

This prospective study involving 2,499 $(1,453$ female and 1,046 male) cohort members of the Framingham Heart Study analyzed the effect of vascular calcification in middle age on participants' subsequent risk of hip fracture. The mean age of participants at the start of the study was 61 years and mean follow-up duration was 21 years, with follow-up consultations scheduled every 2 years. Multivariable statistical models were adjusted for age, BMI, coronary heart disease, diabetes, estrogen use (in women), smoking, systolic blood pressure and total cholesterol. One potential limitation of this study was the analysis of vascular calcification using plain radiographs rather than current technologies, such as CT.

Cumulative incidence of hip fracture did not differ between participants with the absence or presence of vascular calcification, nor did it increase with severity of aortic calcification. Increasing severity of aortic calcification was, however, highly associated with participant mortality. Samelson et al. concluded that the common radiographic finding of aortic calcification should not be used to identify individuals with an increased risk of hip fracture.

Original article Samelson EJ et al. (2007) Vascular calcification in middle age and long-term risk of hip fracture: The Framingham study. J Bone Miner Res 22: 1449-1454

\section{Infliximab does not completely inhibit radiographic progression in patients with AS}

In the open extension of the first randomized controlled trial of infliximab therapy for patients with ankylosing spondylitis (AS), 2-year followup data showed that patients treated with infliximab had less radiographic progression than patients treated with conventional therapies. Baraliakos and colleagues have now published results from follow-up consultations 4 years after the initiation of infliximab treatment.

The 33 trial participants were treated intravenously with infliximab every 6 weeks for 3 years. Patients were then reinfused with infliximab if, and when, clinical relapse occurred; the median time to relapse was 14.6 weeks. Patients who started the study with radiographic damage showed statistically significant worsening of the chronic spinal changes after 4 years $(P=0.003)$ : 13 of 26 patients showed some radiographic changes, with 10 of these patients classified as having definite radiographic deterioration. Patients who started the study with no radiographic damage did not show significant deterioration $(P=0.08)$ : although four of seven patients showed some changes at the final follow-up, only one of these patients was classified as having definite deterioration. Overall, radiographic change was less pronounced for patients treated with infliximab than for patients who participated in a previous cohort study of conventional AS therapy; however, owing to differences in the average disease activities of the two patient groups, larger trials and a different methodology might be required to confirm this finding.

The authors conclude that although infliximab might decelerate radiographic progression in patients with AS, this anti-TNF therapy does not result in complete inhibition of this process.

Original article Baraliakos X et al. (2007) Radiographic progression in patients with ankylosing spondylitis after 4 yrs of treatment with the anti-TNF- $a$ antibody infliximab. Rheumatology (Oxford) 46: 1450-1453

\section{Developing a valid health-related quality of life questionnaire for patients with SLE}

Systemic lupus erythematosus (SLE) is an autoimmune rheumatic disease that affects predominantly women of childbearing age, particularly those with a Chinese, African American/Caribbean or Asian ethnic background. Treatments developed over the last 40 years have reduced mortality levels substantially; however, SLE produces variable symptoms and its course is unpredictable, with potentially devastating effects on an individual's lifestyle. 